\title{
Diversity, Distribution, and Abundance of Plants in Lewoh-Lebang in the Lebialem Highlands of Southwestern Cameroon
}

\author{
B. A. Fonge, ${ }^{1}$ D. J. Tchetcha, ${ }^{1}$ and L. Nkembi $^{2}$ \\ ${ }^{1}$ Department of Botany and Plant Physiology, University of Buea, P.O. Box 63, Buea, Cameroon \\ ${ }^{2}$ Environment and Rural Development Foundation (ERuDeF), P.O. Box 189, Buea, Cameroon
}

Correspondence should be addressed to B. A. Fonge; ambofonge@yahoo.com

Received 24 April 2013; Accepted 26 June 2013

Academic Editor: Rafael Riosmena-Rodríguez

Copyright (C) 2013 B. A. Fonge et al. This is an open access article distributed under the Creative Commons Attribution License, which permits unrestricted use, distribution, and reproduction in any medium, provided the original work is properly cited.

\begin{abstract}
A survey was conducted between October 2010 and June 2011 to determine the diversity, distribution, and abundance of plants in 4 sites of the Lebialem highlands and to relate species diversity and abundance to altitude and soil types. Twelve (12) plots, each of $1 \mathrm{ha}(250 \times 40 \mathrm{~m})$, were surveyed at the submontane and montane altitudes of the sites. One hundred (100) species belonging to 82 genera were identified with the genera Cola and Psychotria being the most represented. Vulnerable species included Guarea thompsonii, Schefflera hierniana, Allanblackia gabonensis, Cyclomorpha solmsii, Vepris trifoliolata, and Xylopia africana. Species such as Xymalos monospora, Tricalysia atherura, and Piptostigma oyemense present in the study area were endemic to Cameroon. Diversity and distribution of plants were affected by parameters such as the altitude and the soil type. Soil analysis revealed that diversity in the study area was affected by the organic carbon, nitrogen, calcium, and the cation exchange capacity of the soil.
\end{abstract}

\section{Introduction}

Biodiversity is the degree of variation of life forms within a given ecosystem, biome, or entire planet [1]. It encompasses all species of plants, animals and microorganisms, the ecosystem, and ecological processes of which they are parts. It is an umbrella term for the degree of nature's variety, including both number and frequency of ecosystems, species, or genes in a given assemblage. Wilson [2] defines biodiversity as the variety of organisms considered at all levels from genetic variants belonging to the same species through arrays of species to arrays of genera, families, and still higher taxonomic levels.

Besides South Africa, Cameroon is the most biologically rich country known to date on the African continent [3]. It encompasses an intricate mosaic of diverse habitats with moist tropical forest dominating the south and south-east and covering $54 \%$ of the country, mountain forest and savannah in the highlands and sub-Sahelian savannah and near desert in the far north [3]. These diverse habitats harbour more than 9,000 species of plants, 160 species of which are endemic. The majority of the endemic taxa are concentrated around
Mount Cameroon and other highland areas. During the last few decades, deforestation of tropical forests areas has accelerated at an alarming rate as extensive areas of forest are being cleared every year [4]. Man affects the forest ecosystem with activities such as agroindustries, shifting cultivation, and hunting. There has been an overwhelming concern about the loss of tropical diversity and an emphasis on the identification of biodiversity hot spots in an attempt to optimize conservation strategies [5].

Diversity studies carried out in Cameroon have covered many parts of the country but left out certain regions despite their richness in plant diversity $[6,7]$. An example of such a region is the Lewoh-Lebang area in the Lebialem Division. Lebialem is located in the southwest region of Cameroon, and it is characterized by a hilly topography with a rich diversity of flora and fauna. This mountain ecosystem has been under serious pressure from the local people. The ecosystem is a centre of high endemism for many taxa (plants, amphibians, mammals, and birds), and its destruction could lead to the local extinction of globally threatened biodiversity (plants, mammals, etc.), watershed destruction, and degradation of 
livelihood systems, property, and lives. The region also holds some of the globally threatened and endemic species such as the critically endangered cross river gorilla, chimpanzee, flying squirrel, endangered Bannerman's Turaco and Banded Wattle-eye, vulnerable Red-headed Picathartes [8]. The study area is part of the Bamboutos Mountain Range which is a stronghold of montane biodiversity. These ecosystems around the Bamboutos Mountain continue to provide valuable goods and services to local people in the region and are an important watershed lodging the tributaries of Manyu River that drain into the cross river. Due to precedent geological and geographical history of these mountain areas and coupled with the high annual rainfall (2000 to $3000 \mathrm{~mm}$ ) and humidity, these areas are perpetually having landslides $[9,10]$. Most of the landslides are as a result of anthropogenic activities of the communities around the mountain [10]. The Nweh people (tribe in the study site) practice slash and burn agriculture with a bimodal annual farming cycle which is entirely dependent on the rain fall patterns that results in frequent landslides [9]. Information on the type and the distributional patterns of plants may help to put in place proper management schemes on biodiversity conservation. This work therefore assesses the diversity, distribution and abundance of plants found in Lewoh-Lebang landscape in Cameroon so as to propose management schemes for biodiversity conservation.

\section{Materials and Methods}

2.1. Study Area. Lebialem is located in the northeastern part of the southwest region of Cameroon (latitudes $5^{\circ} 38^{\prime} \mathrm{N}$ and $5^{\circ} 43^{\prime} \mathrm{N}$ and between longitudes $9^{\circ} 58^{\prime} \mathrm{E}$ and $10^{\circ} 27^{\prime} \mathrm{E}$ ) [11, 12]. Lewoh-Lebang is located between latitudes $5^{\circ} 45^{\prime}$ and $5^{\circ} 47^{\prime} \mathrm{N}$ and longitudes $9^{\circ} 91^{\prime} \mathrm{E}$ and $9^{\circ} 94^{\prime} \mathrm{E}$ and at altitudes ranging from 1456 to $1835 \mathrm{~m}$ (Figure 1). The climate of this region is similar to that of the Cameroon mountain range which is characterized by high winds and low sunshine [8]. The average daily temperature varies very much with seasons but has ranges of 17 to $32^{\circ} \mathrm{C}$, and the mean annual rainfall range from 2000 was $3000 \mathrm{~mm}$ [8]. The main vegetation type is grassland with patches of montane and submontane forests mainly as a result of human activities particularly cocoyam farming in the lowland forest [13].

2.2. Sampling. Sampling was carried out in four different sites: Atullah $\left(5^{\circ} 46^{\prime} \mathrm{N}\right.$ and $\left.9^{\circ} 93^{\prime} \mathrm{E}\right)$, Leleng $\left(5^{\circ} 47^{\prime} \mathrm{N}\right.$ and $\left.9^{\circ} 94^{\prime} \mathrm{E}\right)$, Mbindia $\left(5^{\circ} 45^{\prime} \mathrm{N}\right.$ and $\left.9^{\circ} 91^{\prime} \mathrm{E}\right)$, and Nyitebong $\left(5^{\circ} 46^{\prime} \mathrm{N}\right.$ and $\left.9^{\circ} 91^{\prime} \mathrm{E}\right)$. Each site was divided into submontane (800-1600 m altitude) and montane levels ( $\geq 1600 \mathrm{~m}$ altitude). The study sites and the altitudes used were subdivided following the classification done by Harvey et al. [14]. Within each of the stratum, a plot of $1 \mathrm{ha}(40 \mathrm{~m} \times 250 \mathrm{~m})$ was laid and was subdivided into 10 subplots of $10 \times 10 \mathrm{~m}$ placed at regular intervals of $50 \mathrm{~m}$ from each other. Within each plot, all individual trees were identified, measured, and recorded. Taxa were identified in situ by a taxonomist from the Limbe Botanic Garden. The diameters at breast height of the species were measured using a diameter tape. Trees were grouped into the following diameter classes: small trees $(1-9.9 \mathrm{~cm})$, medium-sized trees $(10-29.9 \mathrm{~cm})$, and large trees $(>29.9 \mathrm{~cm})$ following a grouping done by Kenfack et al. [15]. These species were further grouped into four life forms defined by their maximum attainable heights as follows: treelets (small trees) $(<10 \mathrm{~m})$, understorey $(10-20 \mathrm{~m})$, canopy $(20-30 \mathrm{~m})$, and emergent $(>30 \mathrm{~m})[16]$.

Voucher specimens were prepared and compared with those at the Limbe Botanic Garden Herbarium (SCA) and the Cameroon National Herbarium (YA). Rare species were identified in situ to prevent forward destruction. At each altitude level, soil samples were collected, air dried and standard procedures [17-20] were used to analyse the samples. The following soil parameters were analysed: soil $\mathrm{pH}$ determined in the ration of 2:5 (w/v) soil water suspension, organic carbon by chromic acid digestion and spectrophotometric analysis [18]. Total nitrogen was determined by wet acid digestion [17], and exchangeable cations (calcium, magnesium, and potassium) were extracted using the Mehlich-3 procedure [19] and atomic absorption spectrophotometry. Available phosphorus was extracted by the Bray-1 procedure and analysed using the molybdate blue procedure described by Murphy and Riley [20].

2.3. Data Processing and Analysis. Species diversities were determined using the Shannon-Weaver Diversity Index $(H)$ : $H=(P i)(\log n P i)$, where $P i=n i / N, n i=$ number of individuals of species $i$, and $N=$ total number of individuals [21]. Pearson correlation was conducted to determine the relationship between the soil physicochemical factors and species richness and diversity.

\section{Results}

Table 1 shows the different plant species, their code, authors and life forms found in the study sites (Atullah, Leleng, Mbindia, and Nyitebong). A total of 100 species were recorded in all the four sites belonging to 39 families and 82 genera in which $94 \%$ were identified to species level and $6 \%$ identified to genus level. Out of the 100 species identified in the study sites, 39 species were treelets, mostly $<10 \mathrm{~m}$ tall, and 24 species were understory trees $<20 \mathrm{~m}$ tall and seldom reaching the canopy. Twenty-five (25) species were main canopy species and 11 species were emergent trees species.

From the 39 families recorded in the study sites, the Rubiaceae had the highest number of genera (12) and species (17) followed by the Sterculiaceae with 6 species and 3 genera. A total of 82 genera were recorded in the study sites. Cola (Rubiaceae) were the most abundant genera with the highest number of species (4). This was followed by the genera Strombosia (Olacaceae) and Vernonia (Asteraceae) having 3 species each (Table 1 ).

Table 2 shows the different trees species found in the different study sites and their relative abundance. In the four sites, 2113 individuals were sampled. The species with the highest number of individual was Macaranga monandra (179) with a relative abundance of $8.47 \%$. It was followed by Pentadesma butyracea (131 individuals and relative abundance 


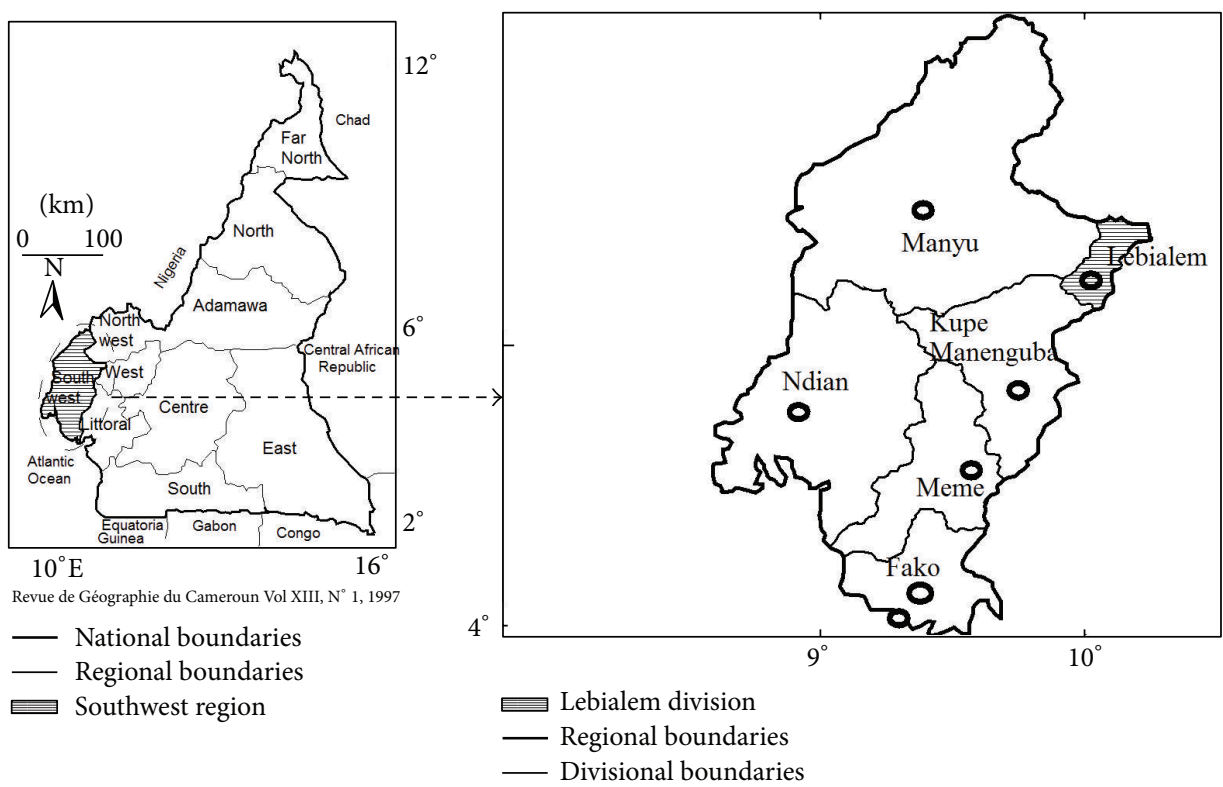

(a) Southwest region in Cameroon

(b) Lebialem division in the southwest region

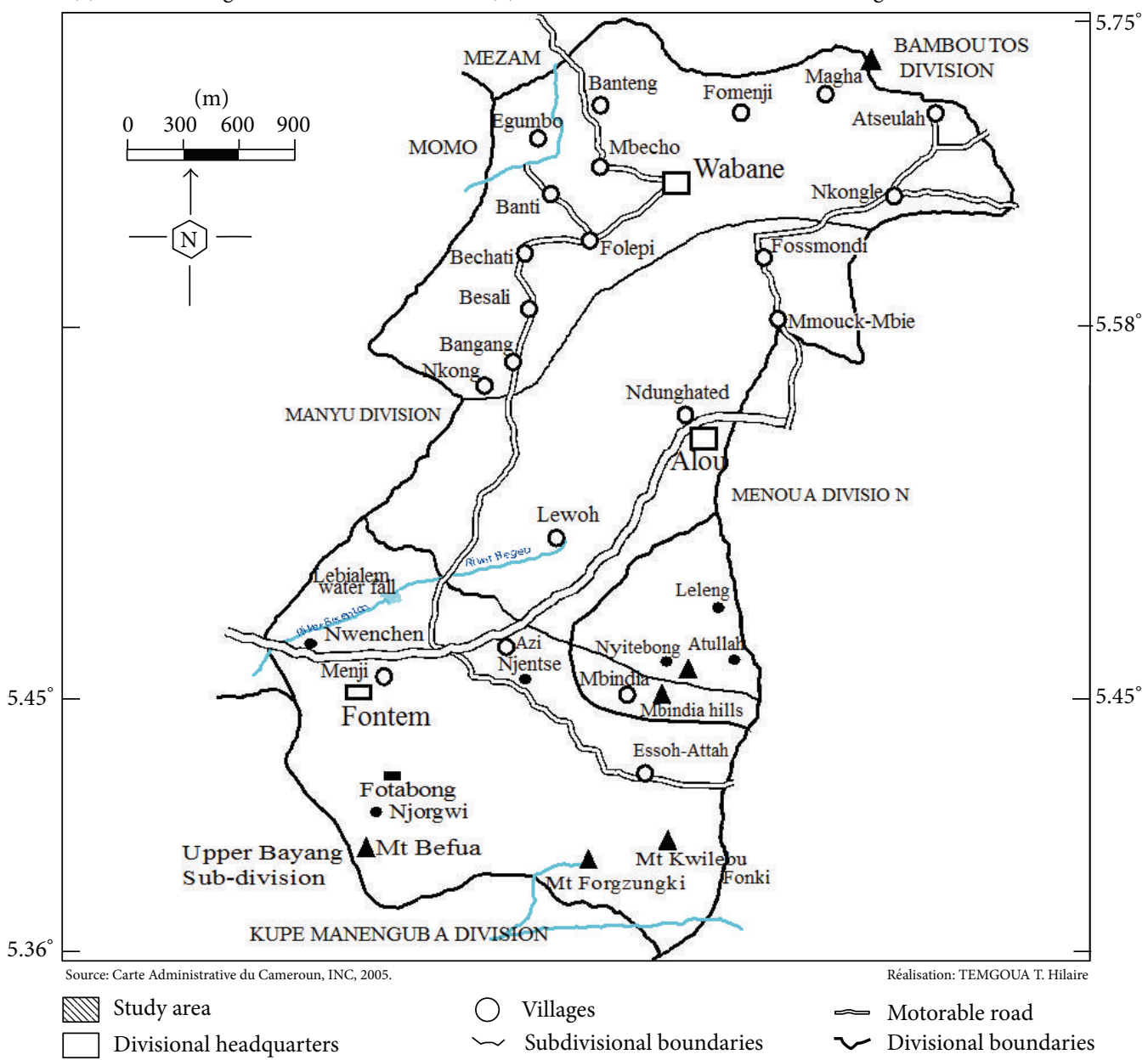

(c) Area of study in Lebialem division

FIgure 1: Map of Atullah, Leleng, Mbindia, and Nyitebong forests, Southwest Cameroon. 
TABLE 1: Species composition of plants at the study sites (Atullah, Leleng, Mbindia, and Nyitebong forests).

\begin{tabular}{|c|c|c|c|c|c|}
\hline Code & Family & Genera & Scientific name & Author(s) & Form \\
\hline CAE2 & Fabaceae & Albizia & Albizia adianthifolia & (Schumach.) W. F. Wight & Canopy \\
\hline ALFL & Clusiaceae & Allanblackia & Allanblackia gabonensis & (Pellegr.) Bamps & Canopy \\
\hline MAL1 & Phyllanthaceae & Antidesma & Antidesma laciniatum & Mull.Arg. & Treelet \\
\hline WARA & Rutaceae & Araliopsis & Araliopsis tabouensis & Aubrev. and Pellegr. & Emergent \\
\hline BEIL & Lauraceae & Beilschmiedia & Beilschmiedia sp. 1 & & Canopy \\
\hline WFIC & Lauraceae & Beilschmiedia & Beilschmiedia sp. 2 & & Understorey \\
\hline BEL1 & Rubiaceae & Belonophora & Belonophora coriacea & Holye & Treelet \\
\hline BEWE & Rubiaceae & Belonophora & Belonophora werhnamii & Hutch. and Dalziel & Treelet \\
\hline WBER & Melianthaceae & Bersama & Bersama abyssinica & Fres & Treelet \\
\hline CPD3 & Sapindaceae & Blighia & Blighia sapida & Konig & Emergent \\
\hline BRMI & Phyllanthaceae & Bridelia & Bridelia micrantha & (Hochst.) Baill. & Canopy \\
\hline WCAR & Meliaceae & Carapa & Carapa grandiflora & Sprague & Canopy \\
\hline COF4 & Rubiaceae & Chazaliella & Chazaliella sp. & & Understorey \\
\hline SAP5 & Chrysobalanaceae & Chrysobalanus & Chrysobalanus icaco & (A. Chev.) F. White & Canopy \\
\hline SAPO & Sapotaceae & Chrysophyllum & Chrysophyllum sp. & & Canopy \\
\hline CPD1 & Sapindaceae & Chytranthus & Chytranthus talbotia & (Baker f.) Keay & Understorey \\
\hline UNKN 2 & Rubiaceae & Coffea & Coffea sp. & & Treelet \\
\hline $\mathrm{COHE}$ & Sterculiaceae & Cola & Cola heterophylla & (P.Beauv.) Schott and Endl & Treelet \\
\hline COME & Sterculiaceae & Cola & Cola megalophylla & Brenan and Keay & Emergent \\
\hline CONI & Sterculiaceae & Cola & Cola accuminata & (Vent.) Schott and Endt. & Understorey \\
\hline MAL2 & Sterculiaceae & Cola & Cola chlamydantha & K. Schum. & Understorey \\
\hline CRAR & Rubiaceae & Craterispermum & Craterispermum aristatum & Wernharm & Treelet \\
\hline CRSP & Rubiaceae & Craterispermum & Craterispermum cf laurinum & (Poir) Benth. & Treelet \\
\hline WRIC & Caricaceae & Cylicomorpha & Cylicomorpha solmsii & (Urb.) Urb. & Treelet \\
\hline DASP & Burseraceae & Dacryodes & Dacryodes klaineana & (Pierre) H.J. Lam & Canopy \\
\hline BAIN? & Flacourtiaceae & Dasylepis & Dasylepis blackii & (Oliv. ) Chipp & Understorey \\
\hline DORT & Melastomataceae & Dichaetanthera & Dichaetanthera africana & (Hook.f.) Jacq.-Fel. & Treelet \\
\hline DIOG & Olacaceae & Diogoa & Diogoa zenkeri & (Endl.) Exell and Mendonta & Canopy \\
\hline DIIT & Ebenaceae & Diospyros & Diospyros iturensis & (Gurke) Letouzey and F. White & Understorey \\
\hline DRA 1 & Dracaenaceae & Dracaena & Dracaena arborea & (Willd.) Link & Treelet \\
\hline DRY & Euphorbiaceae & Drypetes & Drypetes laciniata & Hutch. & Treelet \\
\hline CACTUS & Euphorbiaceae & Euphorbia & Euphorbia desmindi & Keay and Milne-Redhead & Treelet \\
\hline FICU 2 & Moraceae & Ficus & Ficus mucuso & Welw. Ex. Ficalho & Understorey \\
\hline GASP? & Rubiaceae & Gaertnera & Gaertnera paniculata & Benth. & Treelet \\
\hline SAP1 & Sapotaceae & Gambeya & Gambeya africana & G. Don & Understorey \\
\hline PEBU2 & Clusiaceae & Garcinia & Garcinia smeathmanii & (Planch and Triana) Oliv. & Understorey \\
\hline UN4 & Tiliaceae & Glyphaea & Glyphaea brevis & (Spreng.) Monach. & Treelet \\
\hline $\mathrm{CPD} 4$ & Meliaceae & Guarea & Guarea cf glomerulata & Harm & Treelet \\
\hline GUA & Meliaceae & Guarea & Guarea thompsonii & Sprague and Hutch. & Emergent \\
\hline WCRAR & Simaroubaceae & Hannoa & Hannoa klaineana & Pierre and Engl. & Canopy \\
\hline WPYAN & Clusiaceae & Harungana & Harungana madagascariensis & Lam. ex Poir & Understorey \\
\hline HYZE & Lauraceae & Hypodaphnis & Hypodaphnis zenkeri & (Engl.) Stapf. & Canopy \\
\hline ANNL & Annonaceae & Isolona & Isolona maitlandii & Keay & Canopy \\
\hline IXHI & Rubiaceae & Ixora & Ixora hippoperifera & Bremek. & Treelet \\
\hline UN 1 & Bignonaceae & Kigelia & Kigelia Africana & (Lam.) Benth. & Canopy \\
\hline LEKL & Sapotaceae & Lecomtedoxa & Lecomtedoxa klaineana & (Pierre ex Engl.) Dubard & Emergent \\
\hline HOLO & Leeaceae & Leea & Leea guineensis & G. Don & Treelet \\
\hline LEPA & Sterculiaceae & Leptonychia & Leptonychia pallid & K. Schum. & Treelet \\
\hline MAMO & Euphorbiaceae & Macaranga & Macaranga monandra & Mull.Arg. & Canopy \\
\hline ROS & Myrsinaceae & Maesa & Maesa lanceolata & $\mathrm{Mez}$ & Understorey \\
\hline
\end{tabular}


TABLE 1: Continued.

\begin{tabular}{|c|c|c|c|c|c|}
\hline Code & Family & Genera & Scientific name & Author(s) & Form \\
\hline UN2 & Sapotaceae & Manilkara & Manilkara sp. & & Canopy \\
\hline MBETE & Sterculiaceae & Mansonia & Mansonia altissima & (A. Chev.) A. Chev. & Canopy \\
\hline MEM & Melastomataceae & Memecylon & Memecylon afzelii & G. Don & Treelet \\
\hline MIPU & Pandaceae & Microdesmis & Microdesmis puberula & Hook.f. ex Planch. & Treelet \\
\hline MYSP & Cecropiaceae & Myrianthus & Myrianthus preussii & Engl. & Canopy \\
\hline OLA & Olacaceae & Olax & Olax latifolia & Engl. & Treelet \\
\hline COMI & Salicaceae & Oncoba & Oncoba mannii & Oliv. & Understorey \\
\hline ACDET & Rutaceae & Vepris & Vepris trifoliolata & (Engl.) Verdoorn & Treelet \\
\hline SCMA2 & Rubiaceae & Pausinystalia & Pausinystalia macroceras & (K.Schum.) Pierre ex Beille & Canopy \\
\hline PEBU & Clusiaceae & Pentadesma & Pentadesma butyracea & Sabine & Emergent \\
\hline PICA & Piperaceae & Piper & Piper capense & Linn. & Treelet \\
\hline PIAF & Fabaceae & Piptadeniastrum & Piptadeniastrum africanum & (Hook.f.) Brenan & Emergent \\
\hline SCTR & Annonaceae & Piptostigma & Piptostigma oyemense & Pellegrin & Understorey \\
\hline POPA & Annonaceae & Polyceratocarpus & Polyceratocarpus parviflorus & (Baker f.) Ghesq. & Treelet \\
\hline SHAB & Araliaceae & Polyscias & Polyscias fulva & (Hiern) Harms & Understorey \\
\hline PSY & Rubiaceae & Psychotria & Psychotria cf djumaensis & De Wild. & Treelet \\
\hline PSYBM & Rubiaceae & Psychotria & Psychotria penducularis & (Salisb.) Steyerm. & Treelet \\
\hline PSYL & Rubiaceae & Psychotria & Psychotria camptopus & Verdc. & Treelet \\
\hline PSYLS & Rubiaceae & Psychotria & Psychotria strictistipula & Schnell. & Treelet \\
\hline PYAN & Myristicaceae & Pycnanthus & Pycnanthus angolensis & (Welw.) Warb. & Emergent \\
\hline RAVO & Apocynaceae & Rauvolfia & Rauvolfia vomitoria & Afzel. & Understorey \\
\hline ROLU & Rubiaceae & Rothmannia & Rothmannia talbotii & (Wernham) Keay & Treelet \\
\hline WON & Celastraceae & Salicia & Salicia staudtiana & Laos & Understorey \\
\hline SATR & Burseraceae & Santeria & Santeria balsamifera & Oliv. & Emergent \\
\hline OCN & Araliaceae & Schefflera & Schefflera hierniana & Harms & Canopy \\
\hline SCMA & Rubiaceae & Schumanniophyton & Schumanniophyton magnificum & (K.Schum.) Harms & Treelet \\
\hline SPCA & Bignonaceae & Spathodea & Spathodea campanulata & P. Beauv. & Canopy \\
\hline RUBR & Rubiaceae & Stipularia & Stipularia africana & P. Beauv. & Treelet \\
\hline STPU & Olacaceae & Strombosia & Strombosia pustulata & Oliv. & Canopy \\
\hline STRO & Olacaceae & Strombosia & Strombosia grandifolia & Hook.f. ex Benth. & Understorey \\
\hline STSC & Olacaceae & Strombosia & Strombosia scheffleri & Engl. & Canopy \\
\hline GAR2 & Clusiaceae & Symphonia & Symphonia globulifera & L.f. & Emergent \\
\hline TABR & Apocynaceae & Tabernaemontana & Tabernaemontana brachyantha & Stapf & Canopy \\
\hline TACR & Apocynaceae & Tabernaemontana & Tabernaemontana crassa & Benth & Canopy \\
\hline MISP & Ulmaceae & Trema & Trema guineensis & (Schum. and Thonn.) Ficalho. & Understorey \\
\hline $\mathrm{COF}$ & Rubiaceae & Tricalysia & Tricalysia atherura & N. Hallé & Treelet \\
\hline CPD2 & Anacardiaceae & Trichoscypha & Trichoscypha patens & (Oliv.) Engl. & Understorey \\
\hline FICU & Moraceae & Trilepisium & Trilepisuim madagascariense & DC. & Treelet \\
\hline UVKO & Annonaceae & Uvariopsis & Uvariopsis korupensis & Gereau and Kenfack & Understorey \\
\hline VEAM & Asteraceae & Vernonia & Vernonia amygdalina & Del. Cent. & Treelet \\
\hline VECO & Asteraceae & Vernonia & Vernonia conferta & Benth & Treelet \\
\hline VESP & Asteraceae & Vernonia & Vernonia sp. & & Treelet \\
\hline UNKN & Apocynaceae & Voacanga & Voacanga bracteata & Stapf & Treelet \\
\hline VO1 & Apocynaceae & Voacanga & Voacanga psilocalyx & Pierre ex Stapf. & Treelet \\
\hline WAR2 & Melastomataceae & Warneckea & Warneckea jasminoides & (Gilg.) Jacq.-Fel. & Understorey \\
\hline DIOS & Annonaceae & Xylopia & Xylopia africana & (Benth.) Oliv. & Canopy \\
\hline ALPD & Monimiaceae & Xymalos & Xymalos monospora & (Harv.) Baill. Ex Warb. & Understorey \\
\hline WXAN & Rutaceae & Zanthoxylum & Zanthoxylum buesgenii & Engl. & Canopy \\
\hline XANSR & Rutaceae & Zanthoxylum & Zanthoxylum gilletii & (De Wild.) P.G. Waterman & Emergent \\
\hline WAR1 & Melastomataceae & Warneckea pulcherrima & (Hook.f.) Jacq-Fel. & Understorey & \\
\hline
\end{tabular}


TABLE 2: Tree species abundance in the different study sites.

\begin{tabular}{|c|c|c|c|c|c|c|c|c|}
\hline Code & Scientific name & Family & Nyitebong & Mbindia & Attuleh & Leleng & Total & Rel. abun. \\
\hline ACDET & Vepris trifoliata & Rutaceae & - & 7 & - & - & 7 & 0.33 \\
\hline ALFL & Allanblackia gabonensis & Clusiaceae & 1 & - & - & - & 1 & 0.05 \\
\hline ALPD & Xymalos monospora & Monimiaceae & - & - & 54 & 5 & 59 & 2.79 \\
\hline ANNL & Isolona maitlandii & Annonaceae & - & 6 & - & 9 & 15 & 0.71 \\
\hline BAIN? & Dasylepis blackii & Achariaceae & 30 & - & 4 & - & 34 & 1.61 \\
\hline BEIL & Beilschmiedia sp. 1 & Lauraceae & 1 & - & - & - & 1 & 0.05 \\
\hline BEL1 & Belonophora coriacea & Rubiaceae & - & - & 1 & - & 1 & 0.05 \\
\hline BEWE & Belonophora coriacea & Rubiaceae & 1 & 16 & 1 & - & 18 & 0.85 \\
\hline BRMI & Bridelia micrantha & Phyllanthaceae & - & - & 40 & 2 & 42 & 1.99 \\
\hline CACTUS & Euphorbia desmindi & Euphorbiaceae & - & - & - & 1 & 1 & 0.05 \\
\hline CAE2 & Albizia adianthifolia & Fabaceae & - & 7 & - & - & 7 & 0.33 \\
\hline $\mathrm{COF}$ & Tricalysia atherura & Rubiaceae & 1 & 7 & 1 & - & 9 & 0.43 \\
\hline COF4 & Chazaliella sp. & Rubiaceae & 13 & 4 & - & - & 17 & 0.80 \\
\hline COHE & Cola heterophylla & Sterculiaceae & 11 & 46 & - & 1 & 58 & 2.74 \\
\hline COME & Cola megalophylla & Sterculiaceae & 1 & - & - & - & 1 & 0.05 \\
\hline COMI & Oncoba mannii & Salicaceae & 7 & - & - & 1 & 8 & 0.38 \\
\hline CONI & Cola accuminata & Sterculiaceae & - & 1 & 6 & 4 & 11 & 0.52 \\
\hline CPD1 & Chytranthus talbotia & Sapindaceae & 8 & 22 & - & 2 & 32 & 1.51 \\
\hline $\mathrm{CPD} 2$ & Trichoscypha patens & Anacardiaceae & - & - & - & 7 & 7 & 0.33 \\
\hline CPD3 & Blighia sapida & Sapindaceae & - & - & 2 & 2 & 4 & 0.19 \\
\hline CPD4 & Guarea cf glomerulata & Meliaceae & - & - & - & 4 & 4 & 0.19 \\
\hline CRAR & Craterispermum aristatum & Rubiaceae & - & 1 & - & - & 1 & 0.05 \\
\hline CRSP & Craterispermum cf laurinum & Rubiaceae & - & - & - & 2 & 2 & 0.09 \\
\hline DASP & Dacryodes klaineana & Burseraceae & - & 8 & - & - & 8 & 0.38 \\
\hline DIIT & Diospyros iturensis & Ebenaceae & - & 8 & - & - & 8 & 0.38 \\
\hline DIOG & Diogoa zenkeri & Olacaceae & 1 & - & - & - & 1 & 0.05 \\
\hline DIOS & Xylopia africana & Annonaceae & 30 & 1 & 3 & - & 34 & 1.61 \\
\hline DORT & Dichaetanthera Africana & Melastomataceae & - & 6 & - & - & 6 & 0.28 \\
\hline DRA 1 & Dracaena arborea & Dracaenaceae & 16 & 32 & 1 & - & 49 & 2.32 \\
\hline DRY & Drypetes laciniata & Euphorbiaceae & 4 & 12 & - & - & 16 & 0.76 \\
\hline FICU & Trilepisuim madagascariense & Moraceae & 26 & 7 & 4 & 1 & 38 & 1.80 \\
\hline FICU 2 & Ficus mucuso & Moraceae & 11 & 8 & 29 & 18 & 66 & 3.12 \\
\hline GAR2 & Symphonia globulifera & Clusiaceae & - & - & 13 & - & 13 & 0.62 \\
\hline GASP? & Gaertnera paniculata & Rubiaceae & 103 & 2 & 2 & 2 & 109 & 5.16 \\
\hline GUA & Guarea cf thompsonii & Meliaceae & 4 & 3 & - & - & 7 & 0.33 \\
\hline HOLO & Leea guineensis & Leeaceae & - & - & - & 7 & 7 & 0.33 \\
\hline HYZE & Hypodaphnis zenkeri & Lauraceae & - & 5 & - & - & 5 & 0.24 \\
\hline IXHI & Ixora hippoperifera & Rubiaceae & 1 & - & 18 & - & 19 & 0.90 \\
\hline LEKL & Lecomtedoxa klaineana & Sapotaceae & 34 & 6 & - & - & 40 & 1.89 \\
\hline LEPA & Leptonychia pallida & Malvaceae & - & 2 & - & - & 2 & 0.09 \\
\hline MAL1 & Antidesma laciniatum & Phyllanthaceae & - & 7 & - & - & 7 & 0.33 \\
\hline MAL2 & Cola chlamydantha & Sterculiaceae & - & - & 7 & 8 & 15 & 0.71 \\
\hline MAMO & Macaranga monandra & Euphorbiaceae & 15 & 8 & 50 & 106 & 179 & 8.47 \\
\hline MBETE & Mansonia altissima & Malvaceae & - & - & 7 & 2 & 9 & 0.43 \\
\hline MEM & Memecylon afzelii & Melastomataceae & 4 & 1 & - & - & 5 & 0.24 \\
\hline MIPU & Microdesmis puberula & Pandaceae & - & 1 & - & - & 1 & 0.05 \\
\hline MISP & Trema guineensis & Ulmaceae & 1 & - & 12 & 5 & 18 & 0.85 \\
\hline MYSP & Myrianthus preussii & Moraceae & - & 17 & 3 & 7 & 27 & 1.28 \\
\hline $\mathrm{OCN}$ & Schefflera hierniana & Araliaceae & 10 & - & - & - & 10 & 0.47 \\
\hline OLA & Olax latifolia & Olacaceae & 18 & 1 & 1 & - & 20 & 0.95 \\
\hline PEBU & Pentadesma butyracea & Clusiaceae & 123 & 8 & - & - & 131 & 6.20 \\
\hline
\end{tabular}


TABle 2: Continued.

\begin{tabular}{|c|c|c|c|c|c|c|c|c|}
\hline Code & Scientific name & Family & Nyitebong & Mbindia & Attuleh & Leleng & Total & Rel. abun. \\
\hline PEBU2 & Garcinia smeathmanii & Clusiaceae & 2 & - & - & 7 & 9 & 0.43 \\
\hline PIAF & Piptadeniastrum africanum & Fabaceae & - & - & 6 & - & 6 & 0.28 \\
\hline PICA & Piper capense & Piperaceae & - & - & - & 1 & 1 & 0.05 \\
\hline POPA & Polyceratocarpus parviflorus & Annonaceae & - & - & - & 2 & 2 & 0.09 \\
\hline PSY & Psychotria cf djumaensis & Rubiaceae & 5 & 26 & 31 & - & 62 & 2.93 \\
\hline PSYBM & Psychotria peduncularis & Rubiaceae & - & 1 & 29 & 14 & 44 & 2.08 \\
\hline PSYL & Psychotria camptopus & Rubiaceae & 2 & 2 & - & - & 4 & 0.19 \\
\hline PSYLS & Psychotria strictistipula & Rubiaceae & 26 & 19 & 15 & 1 & 61 & 2.89 \\
\hline PYAN & Pycnanthus angolensis & Myristicaceae & 1 & - & - & - & 1 & 0.05 \\
\hline RAVO & Rauvolfia vomitoria & Apocynaceae & - & - & 24 & 20 & 44 & 2.08 \\
\hline ROLU & Rothmannia talbotii & Rubiaceae & - & 4 & - & 1 & 5 & 0.24 \\
\hline ROS & Maesa lanceolata & Myrsinaceae & 46 & 3 & 58 & 2 & 109 & 5.16 \\
\hline RUBR & Stipularia africana & Rubiaceae & 7 & - & - & - & 7 & 0.33 \\
\hline SAP1 & Gambeya africana & Sapotaceae & 1 & 3 & 5 & - & 9 & 0.43 \\
\hline SAP5 & Chrysobalanus icaco & Chrysobalanaceae & - & 2 & 1 & - & 3 & 0.14 \\
\hline SAPO & Chrysophyllum sp. & Sapotaceae & 3 & 1 & - & - & 4 & 0.19 \\
\hline SATR & Santeria balsamifera & Burseraceae & 12 & 22 & - & - & 34 & 1.61 \\
\hline SCMA & Schumanniophyton magnificum & Rubiaceae & 5 & 2 & 8 & - & 15 & 0.71 \\
\hline SCMA2 & Pausinystalia macroceras & Rubiaceae & - & - & 5 & - & 5 & 0.24 \\
\hline SCTR & Piptostigma oyemense & Annonaceae & 1 & - & - & - & 1 & 0.05 \\
\hline SHAB & Polyscias fulva & Araliaceae & - & 2 & - & 12 & 14 & 0.66 \\
\hline SPCA & Spathodea campanulata & Bignonaceae & 1 & - & - & - & 1 & 0.05 \\
\hline STPU & Strombosia pustulata & Olacaceae & - & 17 & 2 & 2 & 21 & 0.99 \\
\hline STRO & Strombosia grandifolia & Olacaceae & 6 & 9 & - & 1 & 16 & 0.76 \\
\hline STSC & Strombosia scheffleri & Olacaceae & 3 & 1 & - & - & 4 & 0.19 \\
\hline TABR & Tabernaemontana brachyantha & Apocynaceae & 2 & - & 1 & 1 & 4 & 0.19 \\
\hline TACR & Tabernaemontana crassa & Apocynaceae & 6 & 8 & 7 & 19 & 40 & 1.89 \\
\hline UN 1 & Kigelia africana & Bignonaceae & 3 & - & - & - & 3 & 0.14 \\
\hline UN2 & Manilkara sp. & Sapotaceae & 6 & 13 & 18 & 3 & 40 & 1.89 \\
\hline UN4 & Glyphaea brevis & Tiliaceae & 9 & 18 & - & - & 27 & 1.28 \\
\hline UNKN & Voacanga bracteata & Apocynaceae & 1 & 2 & - & - & 3 & 0.14 \\
\hline UNKN 2 & Coffea sp. & Rubiaceae & 4 & 2 & 1 & - & 7 & 0.33 \\
\hline UVKO & Uvariopsis korupensis & Annonaceae & 2 & 6 & - & - & 8 & 0.38 \\
\hline VEAM & Vernonia amygdalina & Asteraceae & 3 & - & 4 & - & 7 & 0.33 \\
\hline VECO & Vernonia conferta & Asteraceae & 8 & 9 & 31 & 31 & 79 & 3.74 \\
\hline VESP & Vernonia sp. & Asteraceae & 2 & - & 11 & 1 & 14 & 0.66 \\
\hline VO1 & Voacanga psilocalyx & Apocynaceae & 4 & 3 & - & 1 & 8 & 0.38 \\
\hline WAR1 & Warneckea pulcherrima & Melastomataceae & - & 7 & - & - & 7 & 0.33 \\
\hline WAR2 & Warneckea jasminoides & Melastomataceae & 1 & 6 & 1 & 1 & 9 & 0.43 \\
\hline WARA & Araliopsis tabouensis & Rutaceae & - & 3 & 18 & 9 & 30 & 1.42 \\
\hline WBER & Bersama abyssinica & Melianthaceae & - & - & - & 8 & 8 & 0.38 \\
\hline WCAR & Carapa grandifolia & Meliaceae & 5 & 3 & 22 & 9 & 39 & 1.85 \\
\hline WCRAR & Hannoa klaineana & Simaroubaceae & 24 & 9 & 1 & 1 & 35 & 1.66 \\
\hline WFIC & Beilschmiedia sp. 2 & Lauraceae & 34 & - & 2 & 2 & 38 & 1.80 \\
\hline WON & Salicia staudtiana & Celastraceae & - & - & - & 2 & 2 & 0.09 \\
\hline WPYAN & Harungana madagascariensi & Clusiaceae & 4 & - & 2 & 2 & 8 & 0.38 \\
\hline WRIC & Cylicomorpha solmsii & Caricaceae & 1 & - & - & 2 & 3 & 0.14 \\
\hline WXAN & Zanthoxylum buesgenii & Rutaceae & - & - & - & 7 & 7 & 0.33 \\
\hline XANSR & Zanthoxylum gilletii & Rutaceae & 8 & 1 & 2 & 4 & 15 & 0.71 \\
\hline Total & & & 723 & 464 & 564 & 362 & 2113 & 100 \\
\hline
\end{tabular}


TABLE 3: Diversity indices, evenness, and species richness in different sites.

\begin{tabular}{lccc}
\hline Location & $\begin{array}{c}\text { Shannon } \\
\text { Weaver } \\
\left(H^{\prime}\right)\end{array}$ & $\begin{array}{c}\text { Pielou's } \\
\text { Evenness }(E)\end{array}$ & $\begin{array}{c}\text { Margalef } \\
(D)\end{array}$ \\
\hline Attuleh montane & 2.82 & 0.87 & 4.82 \\
Mbindia submontane & 3.10 & 0.89 & 6.65 \\
Leleng montane & 2.77 & 0.83 & 5.75 \\
Leleng submontane & 2.53 & 0.78 & 4.99 \\
Nyitebong montane & 2.68 & 0.73 & 6.73 \\
Nyitebong submontane & 3.18 & 0.87 & 7.12 \\
\hline
\end{tabular}

of $6.20 \%)$, Gaertnera paniculata, and Maesa lanceolata (109 individuals and relative abundance of 5.16 each).

Thirteen (13) species were common in all the four sites: Trilepisium madagascariense (Moraceae), Ficus mucuso (Moraceae), Gaertnera paniculata (Rubiaceae), Macaranga monandra (Euphorbiaceae), Psychotria strictistipula (Rubiaceae), Maesa lanceolata (Myrsinaceae), Tabernaemontana crassa (Apocynaceae), Manilkara sp. (Sapotaceae), Vernonia conferta (Asteraceae), Warneckea jasminoides (Melastomataceae), Carapa grandiflora (Meliaceae), Hannoa klaineana (Simaroubaceae), and Zanthoxylum gilletii (Rutaceae).

In Nyitebong, 60 species were recorded from 26 families and 51 genera. The most abundant species were Pentadesma butyracea (123 individuals) and Gaertnera paniculata (103) with several families having only one species representative. Allanblackia gabonensis (Clusiaceae), Beilschmiedia sp1 (Lauraceae), Cola megalophylla (Sterculiaceae), Diogoa zenkeri (Olacaceae), Piptostigma oyemense (Annonaceae), Pycnanthus angolensis (Myristicaceae), Spathodea campanulata (Bignoniaceae), and Kigelia africana (Bignoniaceae) were rare species having only one individual recorded in the area.

In Mbindia, 59 species were recorded from 28 families and 51 genera. The most abundant species were Cola heterophylla (46 individuals) and Dracaena arborea (32 individuals). The rare species having only one individual included Craterispermum aristatum (Rubiaceae) and Microdesmis puberula (Pandaceae), which was found only in Mbindia.

In Atullah, 46 species were recorded from 24 families and 51 genera. The most abundant species were Maesa lanceolata (58 individuals) and Xymalos monospora (54 individuals) while Balanophora coriacea (Rubiaceae) was the only rare species.

In Leleng, 49 species were recorded from 28 families and 42 genera. The most abundant species were Macaranga monandra (106 individuals) and Vernonia conferta (31 individuals). The rare species having only one individual and occurring only at Leleng included Euphorbia desmindi (Euphorbiaceae) and Piper capense (Piperaceae).

3.1. Diversity. The Shannon-Weaver Diversity Index $\left(H^{\prime}\right)$, Pielou's Evenness, and the species richness $(d)$ of the different study sites are shown in Table 3. Nyitebong and Mbindia submontane forests were the most diverse communities with

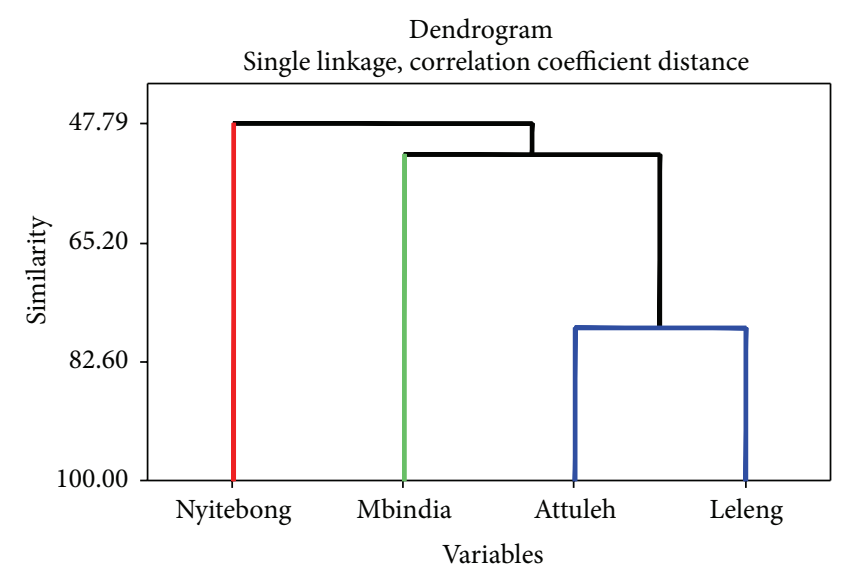

Figure 2: Similarities between the different study sites.

the highest indices of 3.18 and 3.10, respectively. The least diverse sites was Leleng submontane with $H^{\prime}=2.5261$. In terms of evenness, the submontane forest at Mbindia had the most evenly distributed species with Pielou's Evenness value of 0.89 . The richest forest in terms of number of species was the submontane forest at Nyitebong with Margalef richness value of 7.12 .

3.2. Species Similarity. Figure 2 represents a dendrogram showing similarities between the four different study sites. The distance correlation (ward linkage) between Attuleh and Leleng is minimal (0.44), and this shows that Attuleh and Leleng have many plants species that are similar and their similarity index was $77.8 \%$. Nyitebong was less similar to all the other sites.

Figure 3 shows the different diameter classes found in the different study sites. The diameter range was grouped as small trees $(1-9.9 \mathrm{~cm})$, medium-sized trees $(9.9-29.9 \mathrm{~cm})$, and large trees $(>29.9 \mathrm{~cm})$. The four sites were dominated by trees species having diameters ranging from 10 to $99 \mathrm{~mm}$ (small trees). Medium-sized trees were also present in all the sites. There were very few trees with large diameters $(>29.9 \mathrm{~cm})$ occurring at Nyitebong (0.7\%), Mbindia (4.1\%), and Leleng (3.0\%), and no large tree was found in Attuleh forest.

In Nyitebong, 464 trees (82.1\%) with DBH range of $10-$ $99 \mathrm{~mm}$ and 101 trees (17.9\%) with DBH range of 100-299 mm were recorded. In Leleng, 263 trees (72.7\%) with $\mathrm{DBH}$ range of $1-9.9 \mathrm{~cm}, 88$ trees $(24.3 \%)$ with DBH range of $10-29.9 \mathrm{~cm}$, and 11 trees $(3.0 \%)$ with $\mathrm{DBH} \geq 30 \mathrm{~cm}$ were recorded. In Nyitebong, 596 trees (82.1\%) with $\mathrm{DBH}$ range of $1-9.9 \mathrm{~cm}$, 125 trees $(17.2 \%)$ with $\mathrm{DBH}$ range of $10-29.9 \mathrm{~cm}$, and 5 trees $(0.7 \%)$ with $\mathrm{DBH} \geq 30 \mathrm{~cm}$ were recorded. In Mbindia, 379 trees $(81.7 \%)$ with DBH range $1-9.9 \mathrm{~cm}, 66$ trees $(14.2 \%)$ with $\mathrm{DBH}$ range of $10-29.9 \mathrm{~cm}$, and 19 trees $(4.1 \%)$ with $\mathrm{DBH} \geq$ $30 \mathrm{~cm}$ were recorded.

Figure 4 shows the similarities in diameter at breast height between different study sites. It shows that the $\mathrm{DBH}$ of plants in Attuleh and Nyitebong is very similar and that Mbindia has plants with DBH different from that of plants found in all other sites. Trees and shrubs had very similar 
TABle 4: Physicochemical properties of soils at the different sites.

\begin{tabular}{|c|c|c|c|c|c|c|c|c|c|c|c|c|c|c|}
\hline \multirow{2}{*}{ Location/parameter } & \multirow{2}{*}{$\begin{array}{c}\text { Org C } \\
\%\end{array}$} & \multirow{2}{*}{$\begin{array}{c}\mathrm{pH} \\
\text { Water }\end{array}$} & \multirow{2}{*}{$\begin{array}{c}\text { Total N } \\
\% \\
\end{array}$} & \multirow{2}{*}{$\begin{array}{c}\text { Bray } \mathrm{P} \\
\mathrm{ug} / \mathrm{g} \text { or ppm }\end{array}$} & \multicolumn{6}{|c|}{$\operatorname{cmol}(+) / \mathrm{kg}$} & \multirow{2}{*}{$\mathrm{C} / \mathrm{N}$} & \multirow{2}{*}{$\begin{array}{c}\text { Sand } \\
\%\end{array}$} & \multirow{2}{*}{$\begin{array}{c}\text { Clay } \\
\%\end{array}$} & \multirow{2}{*}{$\begin{array}{l}\text { Silt } \\
\%\end{array}$} \\
\hline & & & & & K & $\mathrm{Ca}$ & $\mathrm{Mg}$ & $\mathrm{Na}$ & ECEC & CEC & & & & \\
\hline Atullah montane & 3.73 & 5.19 & 0.42 & 4.00 & 0.49 & 4.86 & 2.04 & 0.04 & 7.44 & 20.58 & 8.83 & 33.57 & 35.89 & 30.53 \\
\hline Mbindia submontane & 3.64 & 4.95 & 0.42 & 2.91 & 0.30 & 3.51 & 1.32 & 0.05 & 5.18 & 18.64 & 8.65 & 32.86 & 33.46 & 33.66 \\
\hline Leleng montane & 2.72 & 5.04 & 0.33 & 2.63 & 0.51 & 3.71 & 1.71 & 0.05 & 5.99 & 19.66 & 8.13 & 32.85 & 40.40 & 26.74 \\
\hline Leleng submontane & 2.61 & 5.32 & 0.32 & 1.91 & 0.30 & 3.91 & 1.43 & 0.04 & 5.68 & 14.59 & 8.00 & 33.54 & 24.04 & 42.42 \\
\hline Nyitebong montane & 5.59 & 5.32 & 0.60 & 2.21 & 0.28 & 0.14 & 0.18 & 0.11 & 0.72 & 21.89 & 9.31 & 57.54 & 14.82 & 27.65 \\
\hline Nyitebong submontane & 4.14 & 4.92 & 0.49 & 6.75 & 0.50 & 3.60 & 1.41 & 0.08 & 5.59 & 25.54 & 8.48 & 51.06 & 24.53 & 24.40 \\
\hline
\end{tabular}

Diameter class distribution for all study sites
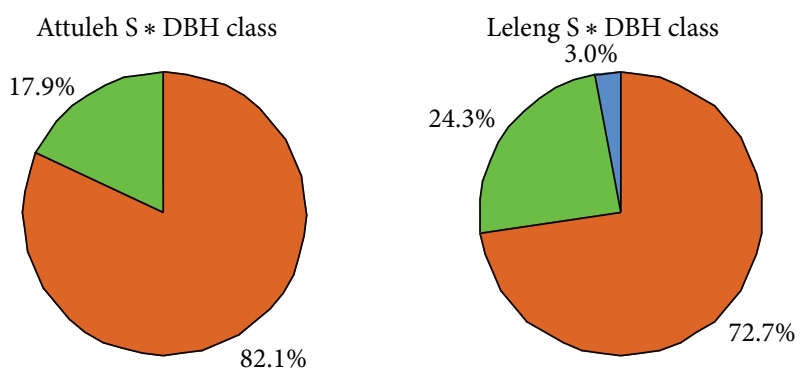

Nyitebong $\mathrm{S} * \mathrm{DBH}$ class

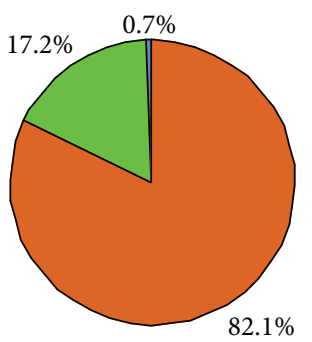

$$
\begin{aligned}
& \text { DBH class } \\
& \square 10-99 \mathrm{~mm} \\
& \square 100-299 \mathrm{~mm} \\
& \square>299 \mathrm{~mm}
\end{aligned}
$$

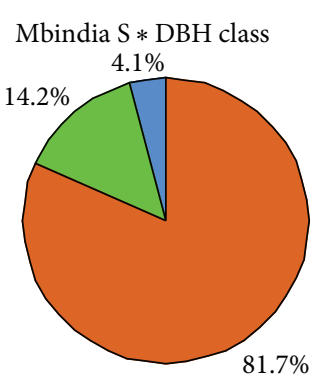

$$
\begin{aligned}
& \text { DBH class } \\
& \square 10-99 \mathrm{~mm} \\
& \square 100-299 \mathrm{~mm} \\
& \square>299 \mathrm{~mm}
\end{aligned}
$$

Figure 3: Diameter class distribution of the different study sites.

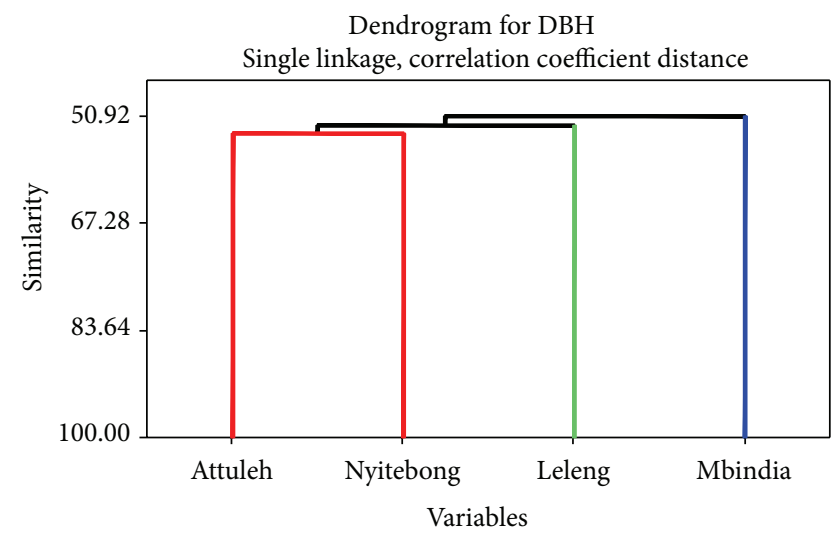

FIGURE 4: Similarities in diameter at breast height between the different study sites. diameters at Attuleh and Nyitebong (53.66). The diameter of plants at Mbindia was different from the diameter of plants in all other areas (50.91).

3.3. Substrate Parameters. Table 4 shows the physico-chemical properties of soils at the different forest levels. The $\mathrm{pH}$ of the study sites was acidic at all the forest levels ranging from 4.92 to 5.22 with Nyitebong submontane being the most acidic site. The calcium $(4.864 \mathrm{cmol} / \mathrm{kg})$, magnesium $(2.043 \mathrm{cmol} / \mathrm{kg})$, and ECEC $(7.444 \mathrm{cmol} / \mathrm{kg})$ content of the soil was higher in Attuleh than in all other sites. The CEC $(25.540 \mathrm{cmol} / \mathrm{kg})$ and Bray $\mathrm{P}(6.750 \mathrm{ppm})$ of the soil were higher in Nyitebong submontane than in all other sites. Nyitebong montane site had the lowest calcium $(0.14 \mathrm{cmol} / \mathrm{kg})$, magnesium $(0.18 \mathrm{cmol} / \mathrm{kg})$, and potassium $(0.28 \mathrm{cmol} / \mathrm{kg})$ content compared with the other sites. The organic carbon $(4.145 \%)$, total nitrogen $(0.601 \%)$, and $\mathrm{C} / \mathrm{N}$ ratios $(9.310 \%)$ of the soil were higher in Nyitebong montane than in all other sites. The soils of Nyitebong montane and submontane sites were sandy having the sand content of 57.40 and $51.06 \%$, respectively. Soils at Attuleh had almost the same soil texture percentages while in Leleng the montane had high clay content $(40.40 \%)$ compared with the submontane with $24.04 \%$ clay and $26.74 \%$ of silt.

Table 5 shows the correlation between soil parameters, diversity indices, index of evenness, and species richness. The diversity and evenness of plants in study sites were negatively correlated with $\mathrm{pH}$ while there was no correlation with species richness $(P>0.01$ and $P>0.05$, resp.). Evenness was positively correlated with calcium and ECEC $(P>0.05)$. The diversity of plants was positively correlated with Bray phosphorus content of the soil $(P>0.05)$.

\section{Discussions}

4.1. Species Diversity in the Study Sites. The forests of southwestern Cameroon are generally known to be rich in species diversity because they are located within the high rainfall zone of the Guinean equatorial tropical forest. Tropical forest contains more than half of the global species diversity, and it is often subjected to increasing anthropogenic pressure which leads to loss of biodiversity [22]. It is also believed that this area formed a Pleistocene refugium during the last glacial 


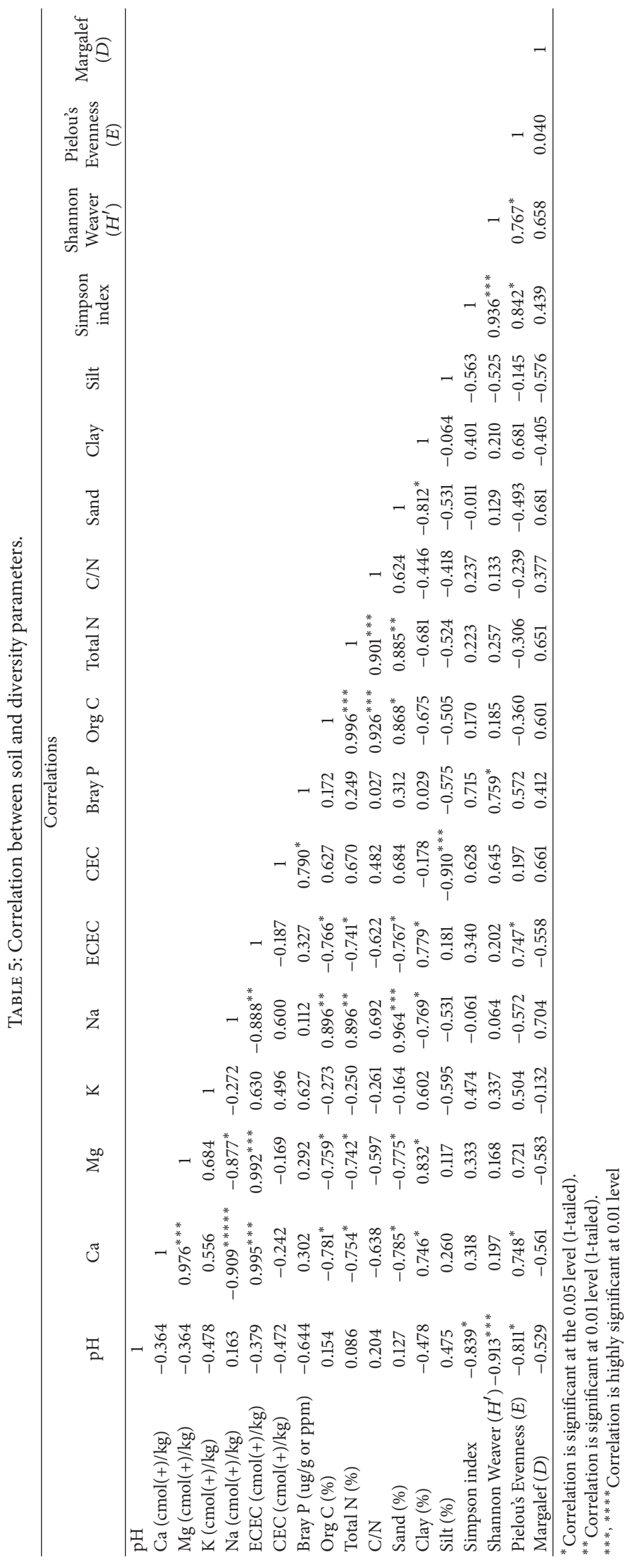


period, becoming isolated and allowing the development of regional endemic species [3].

In the study area, the most dominant family was the Rubiaceae, and this implies that the Rubiaceae could be the most dominant tree family in the Guinean equatorial forest. This result was in line with the findings of Ndam et al. [23] and Fonge et al. [24] who reported that the Rubiaceae was the most dominant tree family in the Mount Cameroon region. Kenfack et al. [15] also report Rubiaceae to be the most dominant tree family in the Korup National Park, and Kouamé et al. [25] reported that the Rubiaceae was the most dominant tree family in the Azagny National Park of Cote D'Ivoire. The submontane forest had more species than the montane forest, and this could be due to the fact that species richness decreases with altitude [3]. Twenty-eight (28) species of plants were found only in the submontane forest, while 15 plant species were found only in the montane forest. This high number of species found in the submontane area could be due to the disturbance (agriculture) which brings about the establishment of secondary species [11]. Thirteen (13) species of plants cut across all the 4 study sites and the ecology of these species show that they thrive across a wide range of habitats including both montane and submontane habitats.

Allanblackia gabonensis is a rare species occurring only at Nyitebong. The absence of this species in the other sites might be due to deforestation. At these sites (Mbindia, Atullah, and Leleng) human activities particularly agriculture (slash and burn farming system) were higher than those in Nyitebong. Allanblackia gabonensis is of particular interest because it is vulnerable and of great economic value. This result is in line with the findings of Ndam et al. [26] who also reported Allanblackia gabonensis to be a rare species in the Mount Cameroon montane forest.

4.2. Species Richness and Diversity. According to Kent and Coker [27], a forest community is said to be rich if it has a Shannon Diversity value $\geq 3.5$. All our sites had ShannonWeaver Diversity indices values below 3.5 making the forest relatively poor in diversity. The submontane forest at Nyitebong was the most diverse and also the most even forest of all the four study sites followed by the submontane forest at Mbindia. This could be due to the fact that forests at Nyitebong and Mbindia were relatively undisturbed through anthropogenic factors such as agriculture and hunting. Secondly it might also be due to the abandonment of farming activities by the peasants and the successional changes in the vegetation as lands had been left to fallow for a very long time in both areas [24]. This had resulted in the reappearance of many plant species in this area. The submontane forest at Leleng was the least diverse of all the sites. This might be due to anthropogenic effects. In the Leleng area, cultivation, hunting, and collection of forest products were the main activities of the local population. Also we observed large plantations of cocoyams cultivated around the forest edges, and this crop is the main staples of the local community around the forest and is also their source of incoming, hence increasing the pressure on the surrounding forest $[12,24]$. The action of the local people has led to untold suffering including homelessness loss of human lives, properties, and forest land, substantial loss of biodiversity, habitats, and loss of income sources leading to extreme levels of poverty $[9,28]$. There is also loss of cultural values and serious degradation of habitats.

4.3. Threatened Species in the Study Sites. The majority of the taxa found in the studied area are of conservation value and importance. They occur mostly in the intricate mosaic of lowland and ridge forest formations, and the ecological fragility and anthropogenic pressure on the montane forest and submontane forest suggest that these ecotypes are of considerable conservation value. Out of the 100 species recorded, 6 species were threatened. These species included Allanblackia gabonensis, Vepris trifoliolata, Schefflera hierniana, Xylopia africana, Guarea thompsonii, and Cyclomorpha solmsii, and these were all vulnerable species according to IUCN [29]. The presence of these species in the study sites could be because this area is within the Mount Cameroon region which is reported to be a centre of biodiversity and endemism in Cameroon [30]. Scholes and Biggs [30] also found that montane forest contains several centre of endemism for birds, mammals, and plants. The floristic composition and the threatened/endangered species found in the IUCN categories show that this area is qualitatively diverse. The occurrence of threatened species in the area might also be due to the accidental nature of the terrain which restricted human activities especially agriculture to areas that were relatively accessible, thus allowing the inaccessible areas to be relatively undisturbed. Some of the threatened species such as Guarea thompsonii, Cyclomorpha solmsii, and Schefflera hierniana were used in the area as timber, medicine, and fencing, respectively, and this could be the reason why these species did not appear in all the study sites. The following species: Xymalos monospora, Tricalysia atherura, and Piptostigma oyemense which are endemic to Cameroon were also recorded in our study area.

4.4. Substrate Parameters. Based on studies of soil properties, phosphorus present in most tropical soils is lacking due to soil acidity, and fixation therefore becomes unavailable to plants for proper growth and development [31].

Forest ecosystems are highly diversified in plant species and this great floristic diversity is supported by relatively poor and acidic soils [1]. Nyitebong was the most diverse of all sites having very acidic soils that have low calcium, magnesium, potassium, and sodium concentrations. This result corroborates the findings of Fonge et al. [24] who reported similar results in soils of the Mount Cameroon region. Nyitebong submontane forest also had the highest values in terms of carbon: nitrogen ratio, organic carbon, total nitrogen, Bray phosphorus, and CEC, and this explains its high floristic diversity. The high content of these elements could be because of the continuous accumulation of organic material on the top soil over the years from pioneer species (bryophytes, ferns, orchids, etc.), litter from trees, shrubs, and dead macro- and microorganisms which could be responsible for the regeneration of the vegetation cover [24, 32]. Nyitebong submontane had the highest percentages of organic carbon, and this might be the reason why they had a greater diversity compared with the other sites. 
Pearson correlation shows that organic carbon was positively correlated with the total nitrogen and the carbonnitrogen ratio. These two nutrients are essential macronutrients for plant growth and vegetation establishment. The humid substances from the decay of organic materials aid in weathering of the parent rock and thereby increasing the amount of silt and clay in the soil. Nevertheless, this was not the case in Nyitebong where the sand content was high and this high content of sand could be attributed to the composition of the parent rock material and the weathering processes involved during soil formation and high rainfall which causes the leaching of nutrients from the soil. Leleng montane forest unlike most montane ecosystems had soils with high clay content. This might be due to the fact that the slope at Leleng was not steep and thus reducing the rate of erosion. Diversity was positively correlated with phosphorus concentration in the soil while it was negatively correlated with the $\mathrm{pH}$. Evenness was negatively correlated with $\mathrm{pH}$ and positively correlated with ECEC and the calcium content of the soil. Potassium did not correlate with any of the parameters meaning that potassium did not influence the diversity and distribution of species in the study area. Phosphorus concentration of the soils (6.75 ppm) was the highest in Nyitebong submontane, but this value was relatively low compared with the findings of Mvondo Ze [33] who reported the phosphorus content of Mount Cameroon soils to be between 12 and $16 \mathrm{ppm}$. The low phosphorus concentration of soils in the study sites might be the reason for the low diversity in the area. Phosphorus was negatively correlated with $\mathrm{pH}$ in our study sites, and this was in line with the findings of Wada and Gunjigake [34] who reported that the amount of phosphorus in soils is correlated with the $\mathrm{pH}$ of the soil.

\section{Conclusion}

Biodiversity is in need of wise management not only to satisfy international pressures and obligations, but also because biodiversity could be the basis of most rural sustainable livelihoods in new economic sectors. The montane and submontane vegetation was subjected to human disturbance. In the Lebialem region, most of the tree species are treelets with a height range of about $<10 \mathrm{~m}$ signifying anthropogenic disturbance. Rubiaceae was the most common family with Cola being the most abundant genera followed by Strombosia (Olacaceae) and Vernonia (Asteraceae). The tree species were greatly affected by the soil physicochemical properties and were positively correlated with Bray phosphorus.

\section{Recommendations}

The population needs to be educated on sustainable farming techniques (e.g., agroforestry that maximizes production in reduced surface area) and sustainable forest management. This will help reduce the pressure on the forest and thus conserving the natural environment.

More research should be geared towards effects of climate and landuse changes factors on vegetation establishment in this area as this will help in the management of landslide activities in these ecosystems.
Reforestation programmes should be carried out by the government and councils to improve the water catchment.

\section{Acknowledgments}

Special thanks go to the University of Buea that gave the initial grant used to carry out this research. The authors gratefully acknowledge the collaboration of the villagers in the Lewoh and Lebang villages in this study as well as the Limbe Botanic Garden and the botanists of the Cameroon National Herbarium for their help in validating the identities of specimens. Also the financial support of the NGO "Environment and Rural Development Foundation" (ERUDEF) is gratefully acknowledged.

\section{References}

[1] G. Uno, R. Storey, and R. Moore, Principles of Botany, McGrawHill, 2001.

[2] E. O. Wilson, The Diversity of Life, Penguin Books, 1992.

[3] T. C. H. Sunderland, J. A. Comiskey, S. Besong, H. Mboh, J. Fonwebon, and M. A. Dione, "Vegetation Assessment of Takamanda Forest Reserve, Cameroon," Smithsonian Institution, 2003.

[4] M. G. P. Tchouto, Plant diversity in a central African rainforest.Implications for biodiversity conservation in Cameroon [Ph.D. thesis], University of Edinburgh/Royal Botanic Garden of Edinburgh, 2004.

[5] H. J. Beentje, Centres of Plant Diversities in Africa, the Biodiversity of African Plants, Kluwer Academic Publishers, The Netherlands, 1996.

[6] J. E. Adjanohoun, N. Aboubakar, K. Dramane et al., "Traditional Medicine and Pharmacopoeia: Contribution to Ethnobotanical and Floristic studies in Cameroon," OAU/STRC, pp. 224-315, 1996.

[7] M. Mbolo, "La collecte et l'analyse des données statistique sur les produits forestiers non ligneux: une étude pilote au Cameroun," Département des forets. In Programme produit forestiers, non ligneux, FAO, Rome, Italie, 2002.

[8] L. Nkembi, "Comparative study of community and government patrols in enhancing sustainable wildlife conservation in the Banyang-Mbo sanctuary, Cameroon," Tech. Rep., Ministry of Environment and Forestry, MINEF, 2004.

[9] S. N. Ayonghe and E. B. Ntasin, "The geological control and triggering mechanism of landslides of the 20th July 2003 with the Bamboutos Caldera, Cameroon," Journal of Cameroon Academic Science, vol. 7, no. 3, pp. 191-203, 2008.

[10] A. Zogning, C. Ngouanet, and O. Tiafack, "The catastrophic geomorphological processes in humid tropical Africa: a case study of the recent landslide disasters in Cameroon," Sedimentary Geology, vol. 199, no. 1-2, pp. 13-27, 2007.

[11] D. A. Focho, E. A. P. Nkeng, B. A. Fonge et al., "Diversity of plants used to treat respiratory diseases in Tubah, northwest region, Cameroon," African Journal of Pharmacy and Pharmacology, vol. 3, no. 11, pp. 573-580, 2009.

[12] D. A. Focho, W. T. Ndam, and B. A. Fonge, "Medicinal plants of Aguambu-Bamumbu in the Lebialem highlands, southwest province of Cameroon," African Journal of Pharmacy and Pharmacology, vol. 3, no. 1, pp. 1-13, 2009. 
[13] B. A. Fonge, E. A. Egbe, A. G. N. Fongod et al., "Ethnobotany survey and uses of plants in the Lewoh-Lebang communities in the Lebialem highlands, South West Region, Cameroon," Journal of Medicinal Plants Research, vol. 6, no. 5, pp. 855-865, 2012.

[14] Y. Harvey, B. Tchieuque, and M. Cheek, “The plants of Lebialem highland, Cameroon," A conservation checklist. Royal botanic Garden, Kew, UK, pp. 7-31 2010.

[15] D. Kenfack, D. W. Thomas, G. Chuyong, and R. Condit, "Rarity and abundance in a diverse African forest," Biodiversity and Conservation, vol. 16, no. 7, pp. 2045-2074, 2007.

[16] G. B. Chuyong, D. Kenfack, K. E. Harms, D. W. Thomas, R. Condit, and L. S. Comita, "Habitat specificity and diversity of tree species in an African wet tropical forest," Plant Ecology, vol. 212, no. 8, pp. 1363-1374, 2011.

[17] A. Buondonno, A. A. Rashad, and E. Coppola, "Comparing tests for soil fertility II. The hydrogen peroxide/sulfuric acid treatment as an alternative to the copper/selenium catalyzed digestion process for routine determination of soil nitrogenKjeldahl," Communications in Soil Science \& Plant Analysis, vol. 26, no. 9-10, pp. 1607-1619, 1995.

[18] D. L. Heanes, "Determination of total organic-C in soils by an improved chromic acid digestion and spectrophotometric procedure," Communications in Soil Science \& Plant Analysis, vol. 15, no. 10, pp. 1191-1213, 1984.

[19] A. Mehlich, "Mehlich 3 soil test extractant: a modification of Mehlich 2 extractant," Communications in Soil Science \& Plant Analysis, vol. 15, no. 12, pp. 1409-1416, 1984.

[20] J. Murphy and J. P. Riley, "A modified single solution method for the determination of phosphate in natural waters," Analytica Chimica Acta, vol. 27, pp. 31-36, 1962.

[21] A. E. Magaurran, Ecological Diversity and Its Measurement, Princeton University Press, Princeton, NJ, USA, 1988.

[22] M. Tchatat, O. Ndoye, and R. NASI, "Produits Forestiers autres que le bois d'oeuvre (PFAB): place dans l'aménagement durable des forêts denses humides d'Afrique Centrale," Projet FORAFRI, 88 pages, 1999.

[23] N. Ndam, J.-P. Nkefor, and P. Blackmore, "Domestication of Gnetum africanum and G. buchholzianum (Gnetaceae), overexploited wild forest vegetables of the Central African Region," Systematics and Geography of Plants, vol. 71, no. 2, pp. 739-745, 2001.

[24] B. A. Fonge, D. A. Focho, E. A. Egbe et al., "The effects of climate and edaphic factors on plant colonisation of lava flows on Mount Cameroon," Journal of Ecology and the Natural Environment, vol. 3, no. 6, pp. 255-267, 2011.

[25] D. Kouamé, Y. C. Y. Abdou, K. E. Kouassi, K. E. N’Guessan, and K. Akoi, "Preliminary floristic inventory and diversity in Azagny National Park (Côte d'Ivoire)," European Journal of Scientific Research, vol. 23, no. 4, pp. 537-547, 2008.

[26] N. Ndam, J. Healey, M. Cheek, and P. Fraser, "Plant recovery on the 1922 and 1959 lava flows on Mount Cameroon, Cameroon," Systematics and Geography of Plants, vol. 71, no. 2, pp. 1023-1032, 2001.

[27] M. Kent and P. Coker, Vegetation Description and Analysis, Belhaven Press, London, UK, 1992.

[28] V. B. Che, M. Kervyn, G. G. J. Ernst et al., "Systematic documentation of landslide events in Limbe area (Mt Cameroon Volcano, SW Cameroon): geometry, controlling, and triggering factors," Natural Hazards, vol. 59, no. 1, pp. 47-74, 2011.
[29] IUCN, Guidelines for Application of IUCN Red List Criteria at Regional Levels: Version 3.0, IUCN Species Survival Commission, IUCN, Gland, Switzerland, 2003.

[30] R. J. Scholes and R. Biggs, Eds., Ecosystem Services in Southern Africa: A Regional Assessment, Council for Scientific and Industrial Research, Pretoria, South Africa, 2004.

[31] P. Vitousek, Nutrient Cycling and Limitation: Hawi'ii as a Model System, Princeton University Press, Princeton, NJ, USA, 2004.

[32] K. Wada, "Allophane and imogolite," in Minerals in Soil Environment, J. B. Dixon and S. B. Weed, Eds., pp. 1051-1087, Soil Science Society of America, Madison, Wis, USA, 2nd edition, 1989.

[33] A. Mvondo Ze, Chemical behaviour of Iron, Manganese Zinc and Phosphorus in selected soils of the Bambouto sequence [Ph.D. thesis], University of Ghent, Gent, Belgium, 1991.

[34] K. Wada and N. Gunjigake, "Active aluminium, iron and phosphate adsorption in andosols," Soil Science, vol. 128, pp. 331-336, 1981. 

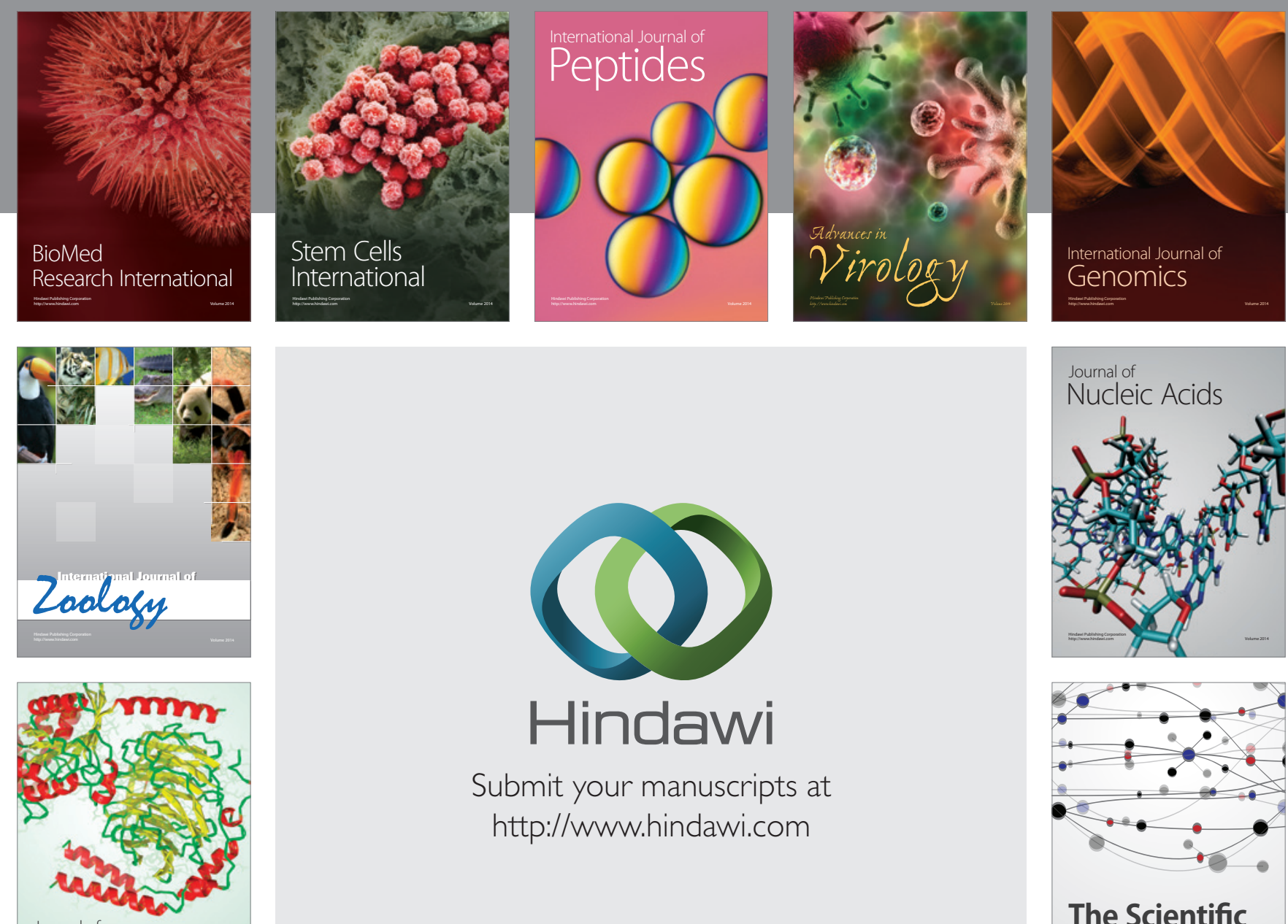

Submit your manuscripts at

http://www.hindawi.com

Journal of
Signal Transduction
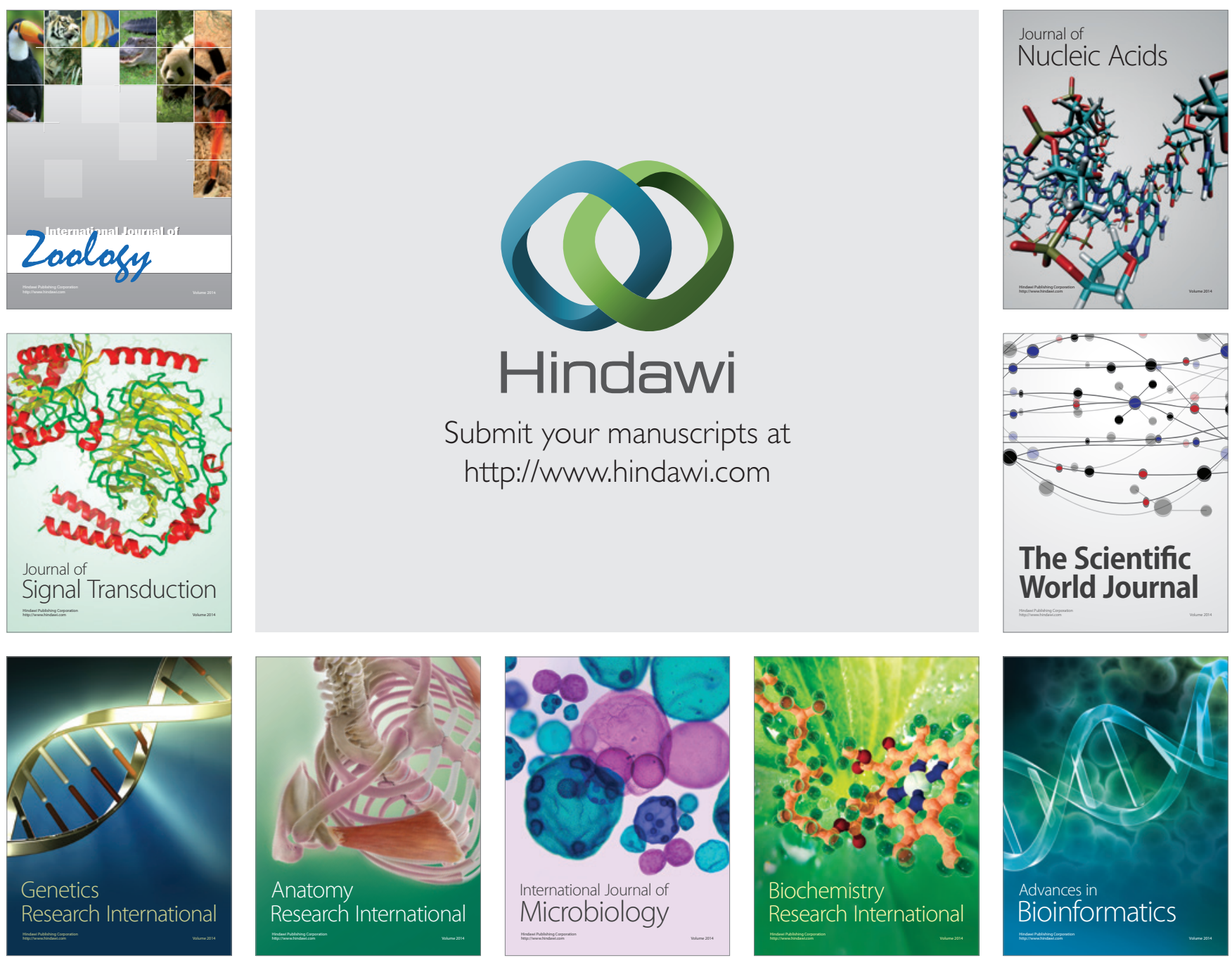

The Scientific World Journal
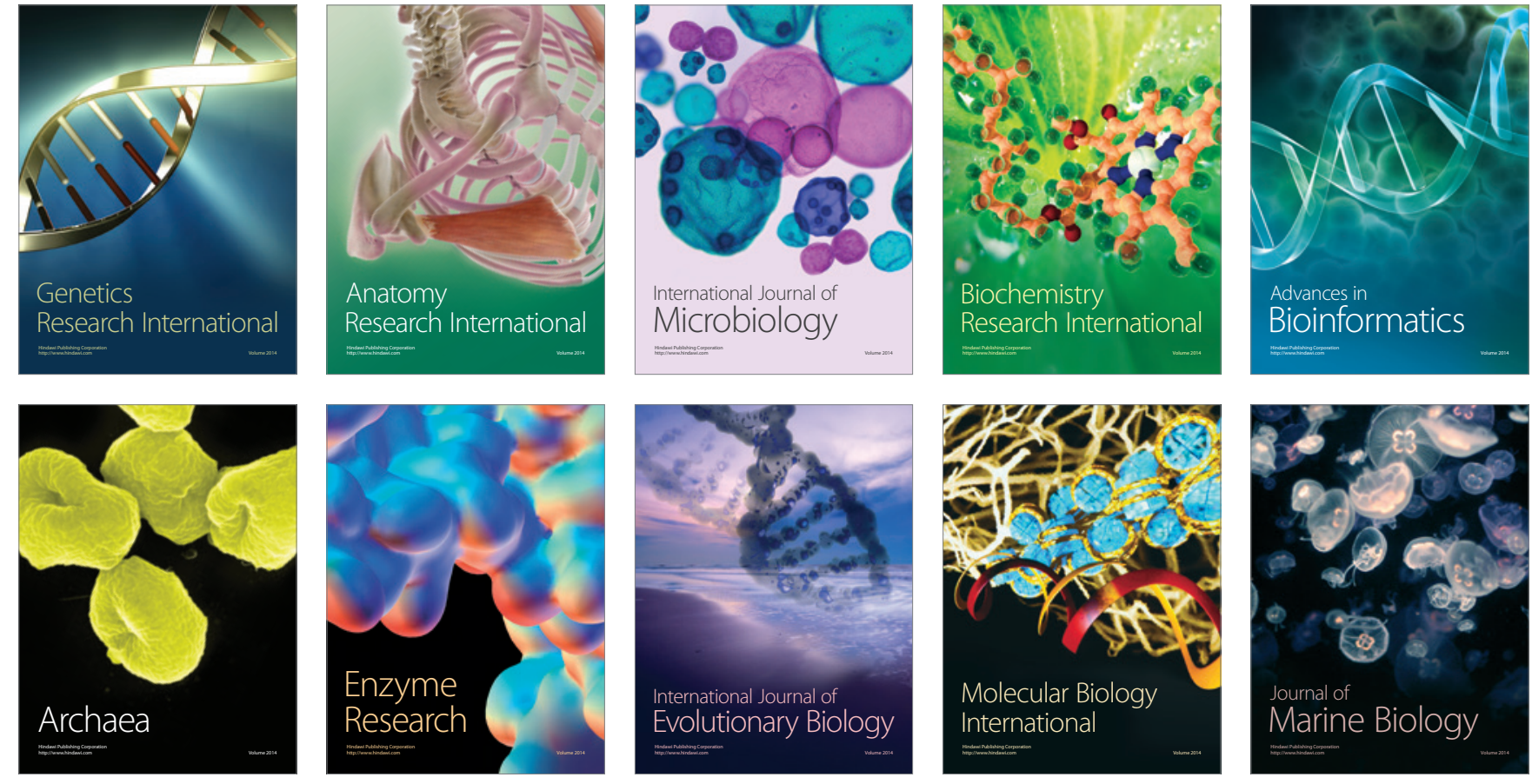